\section{Delayed diagnosed atypical case of Andersen-Tawil syndrome}

\author{
Ahmet Z. Burakgazi \\ Neuroscience Section, Department of \\ Medicine, Virginia Tech Carilion School \\ of Medicine, Roanoke, Virginia, USA
}

\begin{abstract}
Andersen-Tawil syndrome (ATS) is characterized by a triad of periodic paralysis, cardiac arrhythmias and distinctive dysmorphic features. Due to its rarity and high degree of clinical and phenotypic variability, a diagnosis of ATS can be very perplexing and challenging. Herein, an atypical case of ATS with a complicated presentation that caused an approximately 11-year delay in diagnosis is reported. The patient made a full recovery with acetazolamide after the diagnosis. The case and its management are presented with an updated literature review.
\end{abstract}

\section{Introduction}

Andersen-Tawil syndrome (ATS) is characterized by periodic paralysis, cardiac arrhythmia and distinctive features. ATS is a potassium ion channelopathy caused by mutations in the KCJN2 gene. 1,2 This gene encodes the inward rectifier potassium channel, which has a key role in the stabilization of the resting potential of skeletal and cardiac muscles. In contrast to primary periodic paralyses such as hypokalemic or hyperkalemic paralysis which almost exclusively affect skeletal muscles, ${ }^{3}$ ATS affects multiple tissues including the skeletal and cardiac muscles. 3,4

The age of onset of ATS ranges from two to 18 years old. 3,4 The estimated prevalence of ATS is one per one million. ${ }^{5}$ ATS has highly variable clinical presentations with a mean duration of paralysis episodes ranging from one to 26 hours. ${ }^{2,4}$ Patients with ATS do not always have the typical facial features and may have hand and feet peculiarities. They may have long QT intervals and ventricular tachycardia.2,6 Herein, an atypical case of ATS with a complicated presentation that caused an approximately 11-year delay in diagnosis is reported. The patient made a full recovery with acetazolamide after the diagnosis. The case and its management are presented with an updated literature review.

\section{Case Report}

A 28-year-old male was referred to our clinic for the management of periodic paralysis (PP). His symptoms first started at age 16. He first noticed significant weakness when he was in the $10^{\text {th }}$ grade. He had gone hunting and had maintained a squatting position for a prolonged period of time (around 45 minutes). When he woke up the next morning, his legs were extremely weak with no associated pain. The weakness was apparently isolated to his legs. During the first workup, his creatine kinase (CK) level was elevated to approximately 600 units per liter $(\mathrm{U} / \mathrm{L})$. Over the following month, he slowly regained 75 percent of his prior strength. He denied having back pain and had normal bowel and bladder functions. Shortly after the event, he underwent a neurological assessment. He had two normal lower limb nerve conduction studies (NCS) and electromyographies (EMGs), as well as normal repetitive nerve stimulation. A muscle biopsy taken from the thigh muscle was within normal limits. His CK levels ranged from 600-900 U/L. His weakness remained fairly stable after the initial episode. Four months after the first episode, he went to the beach. He had another episode of weakness during that trip. He did not describe any specific triggers such as hot or cold exposure or specific foods such as carbohydrate ingestion. Between the ages of 14 and 20, he experienced periodic lower limb weakness about once a month. Then he had fewer and fewer episodes until age 28 (2018). Between April of 2018 and October of 2018, he experienced more paralytic episodes, and he did not experience full recovery between those episodes. He had significant weakness for a few days. Then, his strength gradually improved, but he did not return to full strength. He did not have any episodes of dark urine.

His past medical history revealed a normal developmental history without any regression. His family history was positive for multiple sclerosis in a paternal uncle and was negative for early muscle weakness and myocardial infarction.

The initial physical examination in December of 2018 showed weakness of the bilateral iliopsoas (based on muscle strength grading 4/5) and the adductor magnus (4/5). The remainder of the patient's neurological examination, including an evaluation of the cranial nerves, a motor exam, a sensory exam and an evaluation of his reflexes, coordination and gait, was within normal limits.

The laboratory investigation results, including L-Carnitine levels and a complete
Correspondence: Ahmet Z. Burakgazi, Neuroscience Section, Department of Medicine, Virginia Tech Carilion School of Medicine, 2 Riverside Circle, Roanoke, VA 24016, USA.

E-mail: drburakgazi@gmail.com

Key words: Andersen-Tawil syndrome; Delayed diagnosed; literature review.

Conflict of interest: the author declares no potential conflict of interest.

Funding: none.

Received for publication: 19 May 2019 Accepted for publication: 11 June 2019

This work is licensed under a Creative Commons Attribution NonCommercial 4.0 License (CC BY-NC 4.0).

(C) Copyright A.Z. Burakgazi, 2019

Licensee PAGEPress, Italy

Neurology International 2019; 11:8180

doi:10.4081/ni.2019.8180

metabolic panel, as well as his cell blood count, erythrocyte sedimentation rate, acylcarnitine profile, anti-nuclear antibodies, and his lactate and thyroid levels, were within normal limits. The CK levels ranged from 400-600 U/L.

He did not take any medication for PP between the ages of 16 and 28 . He initially did not have any cardiac symptoms. The initial electrocardiography (ECG) at age 18 showed multiple ventricular ectopic beats, and the initial echocardiogram was within normal limits. Around age 27, he developed frequent syncope episodes. A cardiac workup showed that his recurrent syncope episodes were related to prolonged QT intervals and polymorphic ventricular tachycardia with deterioration to ventricular fibrillation. A dual chamber, implantable, cardioverter defibrillator was placed which was effective in controlling his syncope episodes. After he developed prolonged QT intervals and ventricular arrhythmia as well as periodic paralysis, a molecular genetic test was performed. The genetic test showed mutations in the KCNJ2 gene. This test was confirmation of a diagnosis of ATS. He was placed on nadolol as well as potassium supplements.

During the neurology visit at age 28, he was put on 250 milligrams (mg) of acetazolamide three times a day. He came back to the neurology clinic after three months for a follow-up visit. He stated that it took a few weeks to see the maximum improvement, but he did not have any more spells, and he 
regained his full strength. He had always experienced partial improvement between the spells (around 75-80 percent), but this marked the first time that he had experienced full recovery in several years. He tolerated acetazolamide with no significant side effects. The follow-up neurological examination, including cranial nerves, a motor exam, a sensory exam, reflexes, coordination and gait, was unremarkable. The follow-up blood work, including a blood cell count, a comprehensive metabolic panel and CK levels, was within normal limits.

\section{Discussion and Conclusions}

Primary PPs are rare, autosomal dominant, genetic neuromuscular disorders linked to mutations in the skeletal muscle ion channels. PPs include hypokalemic paralysis, hyperkalemic paralysis, normokalemic PP, paramyotonia congenita and ATS. The attacks are usually triggered by behavior or diet that may cause changes in serum potassium levels. In our case, the patient did not describe any specific triggers other than the fact that the first attack was triggered after maintaining a prolonged squatting position.

Patients with PP present with episodes of focal or generalized muscle weakness. Patients may or may not experience full recovery between the episodes. The vast majority of patients usually have residual and persistent weakness between the attacks. In our case, the patient did not experience a full recovery between the attacks prior to treatment (he had approximately 75 percent improvement). He almost fully recovered his strength after starting the acetazolamide and did not have any further attacks.

ATS is characterized by a triad of periodic paralysis, cardiac arrhythmias and distinctive dysmorphic features. This characteristic triad is seen in 58-78 percent of ATS patients with KCNJ2 mutations. The first onset of the symptoms usually occurs during the first or second decade of life, with either cardiac symptoms such as palpitations and/or syncope or periodic muscle weakness. The first part of the triad is periodic paralysis. The weakness suddenly takes place after prolonged rest or following rest after exertion. Permanent weakness after the attacks is commonly seen in patients with ATS. The second part of the triad is cardiac arrhythmias including premature ventricular contractions, complex ventricular ectopy, polymorphic ventricular tachycardia (VT), bidirectional VT, and long QTc or long QU intervals. The third part of the triad is dysmorphic features including a small mandible, hypertelorism, a broad nose, syndactyly, clinodactyly and short stature. In our case, the patient's initial symptom was periodic weakness with partial improvement between his attacks at age 16. With the exception of a small mandible, he did not have any of the distinctive dysmorphic features of ATS. The initial ECG was positive for multiple ventricular ectopic beats with no clinical cardiac symptoms. He developed recurrent syncope around age 27. The follow-up EKG showed prolonged QT intervals and polymorphic ventricular tachycardia with deterioration to ventricular fibrillation that necessitated a dual chamber, implantable cardioverter defibrillator placement. The extensive neurological workup, including blood tests, multiple EMGs, and a muscle biopsy, was inconclusive. After the patient developed cardiac symptoms, the clinical picture became clearer, and a diagnosis of ATS was confirmed with genetic testing. Due to its rarity and high degree of clinical and phenotypic variability, a diagnosis of ATS can be very perplexing and challenging.

Genetic testing should be ordered as a first diagnostic step in cases characterized by high clinical suspicion. KCNJ2 gene mutations are identified in around 60 percent of the patients with ATS. No genetic mutations are detected in the rest of the cases (around 40 percent). In ATS cases with the KCNJ2 mutation, 65 percent of the patients have hypokalemic episodes of paralysis, 20 percent have normokalemic episodes, and 15 percent have hyperkalemic episodes. In our case, the KCNJ2 mutation was detected, and no potassium abnormality was detected after the attacks.

Electrodiagnostic testing can be helpful in the diagnosis of PP because commercial genetic testing of PP is incomplete, and insurance coverage of genetic testing is not available in some cases. Regular nerve conduction studies and repetitive studies with short exercise are usually unremarkable. The long exercise test (LET) is a useful test in patients with PP. The LET is typically conducted by stimulating the ulnar nerve at the wrist and recording the adductor digiti minimi motor responses. Different protocols for the LET are described.7-10 The LET shows a post exercise drop in motor response amplitude or area. In one study of ATS, the sensitivity of the LET was between 80 percent and 100 percent. ${ }^{7} \mathrm{~A}$ needle EMG may show positive sharp waves and myotonia (spontaneous waxing and waning of motor unit potentials) in patients with PP.9,11 In our case, two regular NCS and repetitive studies were normal, but the LET protocol was not performed. When our patient was seen in the clinic, the diagnosis was confirmed with genetic testing, so the LET was not performed. The needle EMG was unremarkable as well.

An abnormal muscle biopsy can be seen in patients with periodic paralysis, particularly in hypokalemic periodic paralysis. ${ }^{12,13}$ A muscle biopsy obtained from patients with PP may show myopathy signs including muscle fiber size variability, split fibers, internal nuclei and muscle fiber atrophy. In our case, the muscle biopsy was within normal limits. ${ }^{12,13}$

The management of ATS includes acute and chronic management. During acute management, mild exercise at the onset of the attack, carbohydrate snacks (if ATS is related to hyperkalemia), and potassium supplements (if ATS is related to hypokalemia) may help to alleviate the symptoms. ${ }^{3}$ For prevention, the patient should avoid triggers such as high-carbohydrate and/or high-salt meals, alcohol and stress. Acetazolamide and dichlorphenamide can be effective for chronic treatment. ${ }^{14-18}$ No randomized, controlled studies of acetazolamide in PP have been conducted; the current recommendation is based on nonrandomized, single-blind trials and anecdotal reports. PP subtypes may improve with acetazolamide, but PP subtypes with different gene mutations have different responses to acetazolamide. Dichlorphenamide is approved by the Food and Drug Administration for the treatment of PP.3,17,18 Acetazolamide-related side effects include paresthesia, fatigue, cognitive disturbance and nephrolithiasis ${ }^{14}$ (longterm). Our case was treated with acetazolamide, and he experienced a full recovery with no remarkable side effects.

In conclusion, ATS is a very rare PP with highly variable clinical presentations. The diagnosis can be very challenging. A neuromuscular specialist should be familiar with atypical presentations of PP, particularly ATS, because ATS has a high degree of clinical and phenotypic variability. The skeletal or cardiac findings of ATS may present in a different order and time which may cause a delay in an accurate diagnosis. Further testing, particularly genetic testing, should be ordered in cases with a high degree of clinical suspicion in order to confirm the diagnosis. Avoiding triggers, as well as taking acetazolamide and potassium supplements, are the mainstay treatments of ATS.

\section{References}

1. Bendahhou S, Donaldson MR, Plaster NM, et al. Defective potassium channel 
Kir2.1 trafficking underlies AndersenTawil syndrome. J Biol Chem 2003;278:779-85.

2. Smith AH, Fish FA, Kannankeril PJ. Andersen-Tawil syndrome. Indian Pacing Electrophysiol J 2006;6:32-43.

3. Statland JM, Fontaine B, Hanna MG, et al. Review of the Diagnosis and Treatment of Periodic Paralysis. Muscle Nerve 2018;57:522-30.

4. Veerapandiyan A, Statland JM, Tawil R. Andersen-Tawil Syndrome. Adam MP, Ardinger HH, Pagon RA, et al., eds. Seattle, Washington, 1993.

5. Sansone V, Tawil R. Management and treatment of Andersen-Tawil syndrome (ATS). Neurotherapeutics. 2007;4:3237.

6. Donaldson MR, Yoon G, Fu YH, Ptacek LJ. Andersen-Tawil syndrome: a model of clinical variability, pleiotropy, and genetic heterogeneity. Ann Med 2004;36:92-7.

7. Tan SV, Matthews E, Barber M, et al. Refined exercise testing can aid DNAbased diagnosis in muscle chan- nelopathies. Ann Neurol 2011;69:32840.

8. Fournier E, Arzel M, Sternberg D, et al. Electromyography guides toward subgroups of mutations in muscle channelopathies. Ann Neurol 2004;56:65061.

9. Fournier E, Viala K, Gervais H, et al. Cold extends electromyography distinction between ion channel mutations causing myotonia. Ann Neurol 2006;60:356-65.

10. Kuntzer T, Flocard F, Vial C, et al. Exercise test in muscle channelopathies and other muscle disorders. Muscle Nerve 2000;23:1089-94.

11. Fontaine B. Periodic paralysis. Adv Genet 2008;63:3-23.

12. Weller RO, McArdle B. Calcification within muscle fibres in the periodic paralyses. Brain 1971;94:263-72.

13. Martin JJ, Ceuterick C, Mercelis R, Amrom D. Familial periodic paralysis with hypokalaemia. Study of a muscle biopsy in the myopathic stage of the disorder. Acta Neurol Belg
1984;84:233-42.

14. Tawil R, Moxley RT, Griggs RC. Acetazolamide-induced nephrolithiasis: implications for treatment of neuromuscular disorders. Neurology 1993;43: 1105-6.

15. Matthews E, Portaro S, Ke Q, et al. Acetazolamide efficacy in hypokalemic periodic paralysis and the predictive role of genotype. Neurology 2011;77:1960-4.

16. Platt D, Griggs RC. Use of acetazolamide in sulfonamide-allergic patients with neurologic channelopathies. Arch Neurol 2012;69:527-9.

17. Tawil R, McDermott MP, Brown R Jr, et al. Randomized trials of dichlorphenamide in the periodic paralyses. Working Group on Periodic Paralysis. Ann Neurol 2000;47:46-53.

18. Sansone VA, Burge J, McDermott MP, et al. Randomized, placebo-controlled trials of dichlorphenamide in periodic paralysis. Neurology 2016;86:1408-16. 